\title{
STRUCTURAL AND ELECTRICAL TRANSPORT PROPERTIES OF SnTe FILMS
}

\author{
S.B. Youssef, S.S. Fouad \\ Faculty of Education, Ain Shams University, Cairo, Egypt \\ G. Al-Ganainy and Berlant A. Khalifa \\ Faculty of Education, Ain Shams University, Cairo, Egypt
}

\begin{abstract}
:
Structure investigation of SnTe films, thermally deposited in vacuum of $10^{-5}$ Torr onto glass substrates at room temperature, shows polycrystalline nature of ECC structure. The calculated crystallite size increases with increasing the film thickness. Electrical resistivity $(\rho)$, thermoelectric power $(S)$ and Hall coefficient $\left(R_{H}\right)$ were measured as a function of the film thickness (d). The thickness dependence of the thermoelectric power shows size effect. $\left(R_{H}\right)$ increases with increasing $(d)$. Both thermoelectric power and Hall coefficient measurements show that SnTe films are ntype semiconductors. The free charge carrier concentration $(n)$ is calculated from both measurements and is found to have the same order of magnitude $\left(10_{20} \mathrm{~cm}^{-3}\right)$.
\end{abstract}

\section{INTRODUCTION :}

Tin telluride is one of the important materials of the IV-VI compound for their applications as infrared detectors[1], radiation receivers[2] and in thermoelectronic devices[3]. The transport properties of SnTe single crystals and epitaxial films have been analysed by several workers[4-8]. The influence of the deposition conditions of SnTe thin films grown on different substrates by different techniques on their structural, galvanomagnetic and optical properties have also been thoroughly investigated[9-12]. Despite the great amount of work on the physical properties of $p$ type conductivity of SnTe thin films, a limted data of SnTe properties as n-type semiconductor, in thin film form, are reported [13-15]. In the present study, the electrical resistivity $(\rho)$, the thermoelectric power $(S)$ and the Hall coefficient $\left(R_{H}\right)$ have been measured for n-type SnTe as a function of the film thickness. 


\section{EXPERIMENTAL PROCEDURE :}

Tin telluride material was synthesized by melting the appropriate amounts of the pure components ( 99.999 purity) in evacuated and sealed silica tube at $900^{\circ} \mathrm{C}$ for 6 hours in an oscillatory furnace. The temperature was raised in steps to reduce the vapour pressure of the consitituents. The long duration of the synthesis and the skaking of the molten via the oscillations of the furnance were necessary to ensure the homogeneity of the prepared material. The furnace temperature was reduced gradually to the room temperature by a rate of $20^{\circ} \mathrm{C} /$ hour.

Thin SnTe films of different thinkness have been prepared in vacuum of $3 \times 10^{-5}$ Torr, by conventional thermal evaporation technique, onto the chemically and ultrasonically cleaned glass substrate held at room temperature during the deposition process. The film thickness was controlled using quartz thickness monitor and then measured using multiple beam fizeau fringes [16].

The X-ray diffraction (XRD) of SnTe films were carried out using Philips ciffractometer, with a scanning speed of $2^{\circ}$ minute and scanning angular range of $10^{\circ}$. $80^{\circ}$.

The electrical resistivity $(\rho)$ of the samples was measured by the two-probe technique using a high sensitive digital multimeter (type DA 8601) and a Univeka (type 141) URAN voltmeter.

The thermoelectric power of the samples has been measured relative to copper, by the integral method, in the temperature range of $140-360 \mathrm{~K}$, at a vacuum of $10^{-5} \mathrm{Torr}$. A very thin copper layer, electro-chemically deposited over a silver printed electrodes on the substrates, was used for the thermoelectric power and Hall voltage measurements.

Hall voltage $\left(\mathrm{V}_{\mathrm{z}}\right)$ was measured by a d.c. voltmeter and Tinsley potentiometer (type UJ33E, with an accuracy $\pm 0.5 \mu \mathrm{V}$ ), has been used as null detector. Hall coefficient $\left(R_{H}\right)$ was calculated using the relation $R_{H}=\left(V_{Z} \cdot W\right) /\left(I_{X} \cdot H_{y} \cdot 10^{-8}\right)$ where $W$ 


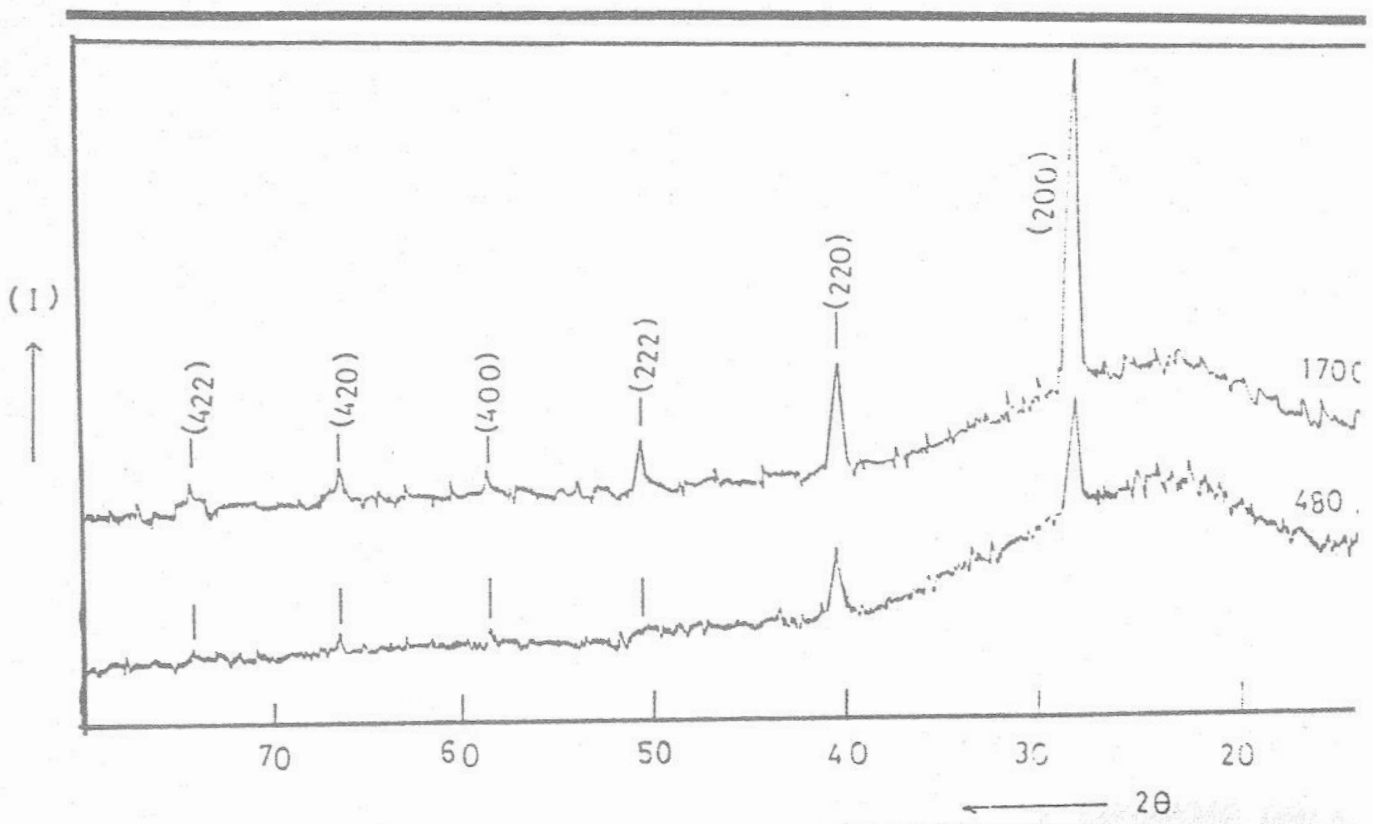

Fig. 1 :

$\mathrm{X}$-ray diffraction pattern of SnTe film of thicknesses $480 \AA$ and $1700 \AA$.

1-b Electrin microscope diffraction pattern of SnTe film of thickness 480A.

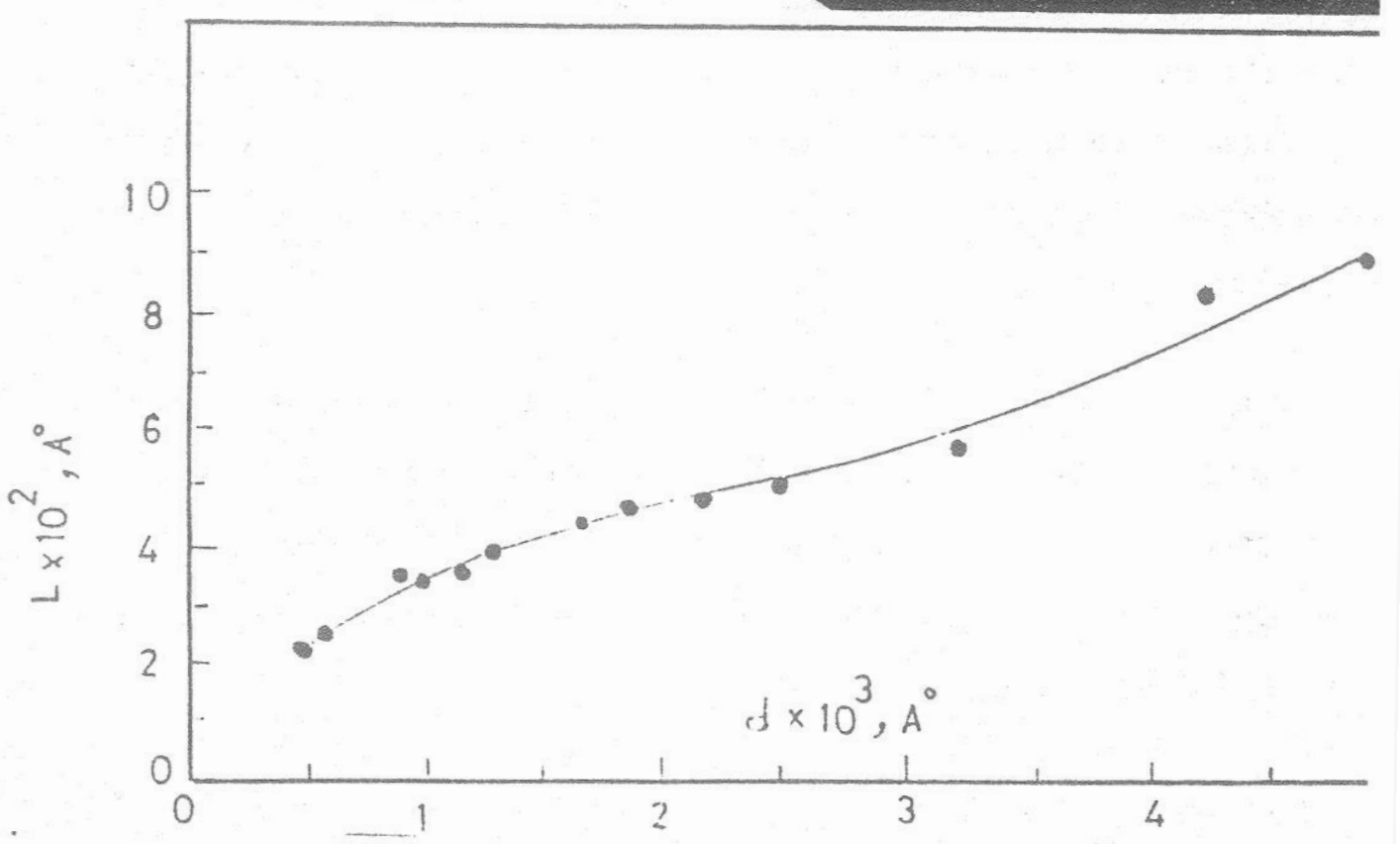

Fig. 2 : a) The effect of the film thickness (d) on the calculated crystallite sia (L) of SnTe films. 
is the width of the film in centimeter, Is the current in amperes and $\mathrm{H}_{\mathrm{y}}$ is the magnetic field in Oersted.

The charge carrier concentration (n) has been calculated from both the Hall coefficient and thermoelectric power measurements. The mobility $(\mu)$ of charge carrier was then calculated according to the relation $\mu=1 /$ e.n.p, where $(n)$ is calculated from Hall coefficient.

\section{RESULTS AND DISCSSION}

\section{Film Structure :-}

The X-ray diffraction (XRD) analysis were carried out for SnTe thin films of different thickness, ranging from $480 \mathrm{~A}^{\circ}$ to $4910 \mathrm{~A}^{\circ}$. Fig.(1-a) illustrates the XRD patterns for films of the thickness 480 and $1700 \mathrm{~A}^{\circ}$ respectively. As the film thickness increases, the intensities of the XRD peaks also increase. All the diffractograms have proven that SnTa Films are polycrystalline and exhibit FCC structure, which also have been confirmed through out the Electon Microscopy analysis (Fig. 1-b). Santhanam et al. [17] have found that SnTe films, deposited onto glass substrates show noncrystalline structure, while those deposited on mica substrates are polycrystalline. However, in another study carried out by the same authers [18], they observed that an optimum substrate temperature $(448 \mathrm{~K})$, the films have crystalline structure weather the substrate was mica or glass.

To compute the individual crystallite size, the $\mathrm{X}$-ray profiles normal to (200) plane, were recorded with a scanning speed $0.125^{\circ} / \mathrm{min}$., through the angular range $27^{\circ}$ $29^{\circ}$. The crystallite size was calculated from the integral breadth according to Scherrer's equation[19]; The integral breadth was corrected for instrumental broadening by Fourrier method.

The calculated crystallite size for various thicknesses of SnTe thin films are illustrated in Fig.(2). This figure shows that the crystallite size increases with increasing the film thickness which reflects the existance of a size effect phenomenan. 


\section{The Electrical Resistivity Measurements :}

The resistivity $(\rho)$ of $\mathrm{SnTe}$ as a function of film thickness (d), at room temperature is shown in Fig.(3a); Where ( $\rho$ ) decreases with increasing (d); this decrease, as a usual behaviour, is due to the size effect which has been discussed through $\mathrm{XRD}$ results. Similar observation of the resistivity dependence on size effect was found earlier [20-22].

Also one can't ignore the lattice defects, such as vacancies, interstitials and dislocations, which may be introduced through the film growth. These defects diffuse and the corresponding resistivity reduces as the film thickness increases.

\section{3 - The Thermoelectric Power Measurements : \\ The coefficient of abosolute thermoelectric power $\left(S_{f}\right)$, for all the studied} samples, was found to be negative, i.e., SnTe thin films are n-type semiconductors. Athwal et al. [13] in their study of the transport properties of $\mathrm{Sn}_{1-\mathrm{x}} \mathrm{Te}_{\mathrm{x}}(0<\mathrm{x}<1)$ alloys found that, thin films evaporated onto glass substrate, by a flash evaporation technique, have n-type conductivities for composition was p-type. The type of conductivity of SnTe may be explained on the basis of cluster calculations of the defects of lattice vacancies (cation or anion vacancies) by Hemstreet [14]. In his study on $\mathrm{SnTe} \& \mathrm{PbTe}$, using the $\mathrm{X} \alpha$ scattered-wave method, he concluded that each anion vacancy porduces two electrons outside of a filled valance band, leading to the n-type behaviour, while each cation vacancy is found to yield two holes in the valance band, producing a p-type behaviour. This indicates that our samples may contain anion vacancies which are responsible for the observed n-type conductivity. Also Mikolaichuk et al. [15] produced epitaxial films of SnTe, on $\mathrm{NCI} \& \mathrm{KBr}$ single crystals, and polycrystalline films, on Zapon, and both have n-type conductivity.

The temperature dependence of the coefficient of the absolute thermoelectric power $\left(\mathrm{S}_{\mathrm{f}}\right)$ of tin telluride films of different thicknesses, has been found to increase with increasing temperature and is shown in Fig. (4). Fig. (3b) depicts the thickness inumntumno of (C) for filme at $\mathrm{rnnm}$ temnerature. As long as the film thickness 
Fig. 3 :

The relation, at $3000 \mathrm{~K}$, between $\mathrm{SnTe}$ films thickness (d) and :

a) the resistivity ( $\rho$ ).

b) the thermoelectric power $\left(\mathrm{S}_{\mathrm{p}}\right)$.

C) the Hall coefficient $\left(R_{H}\right)$.
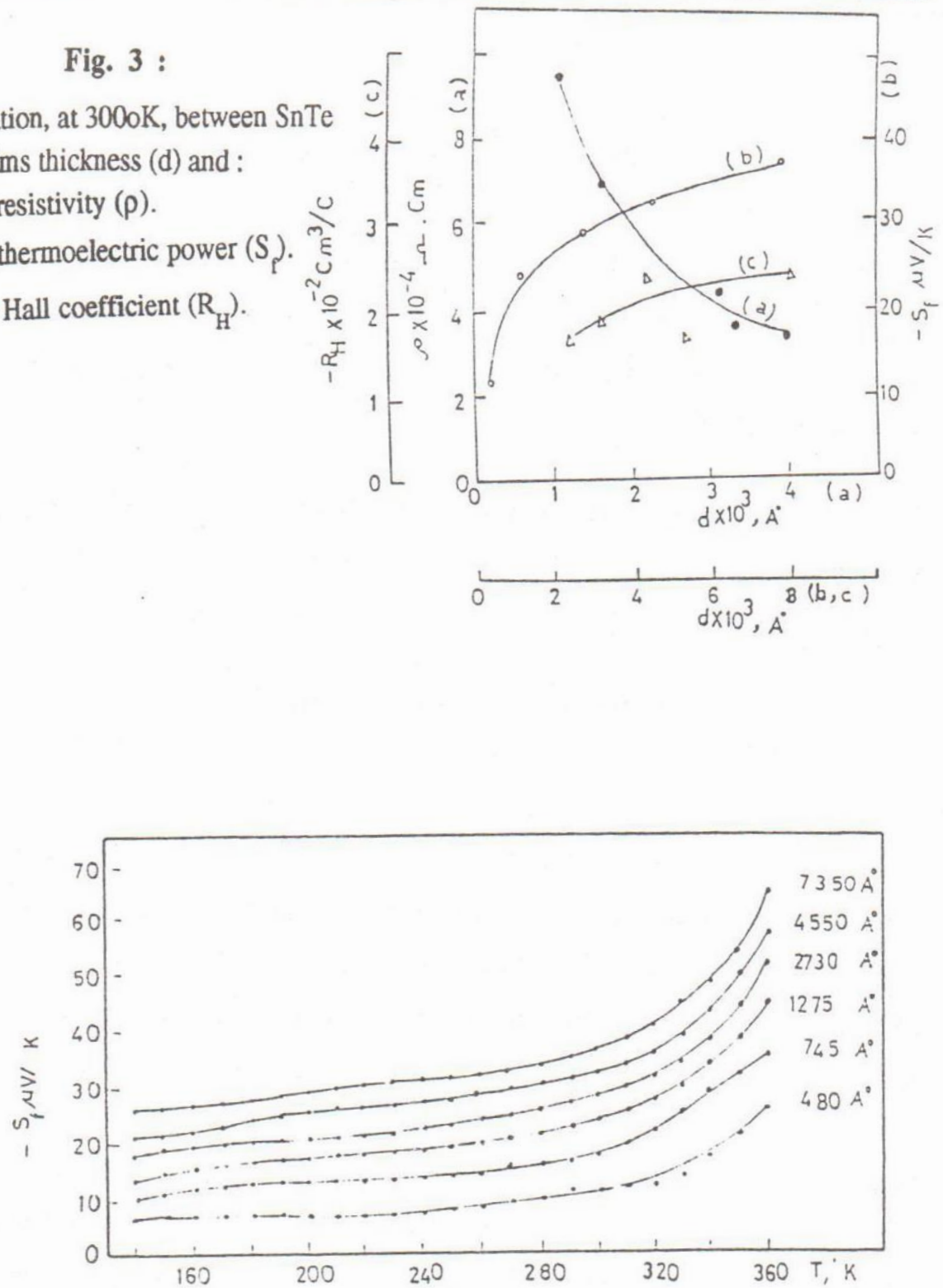

Fig. 4: The relation between the thermoelectric power $\left(S_{f}\right)$ and the corresponding values of temperature $(\mathrm{T})$ for different thicknesses. 
increases, the thermoelectric power increases. From figure (3b), the thickness dependence of the thermoelectric power also shows size effect.

The charge carrier concentration $(n)$ has been calculated from the thermoelectric power data by using the equations (23] :

$$
S_{f}=\left(E_{c}-E_{f}\right)+\frac{2 k_{B} T}{e}
$$

and

$$
\mathrm{n}=2\left(2 \pi \mathrm{m} *\left(\mathrm{k}_{\mathrm{B}} T \mathrm{~h}^{2}\right)^{3 / 2} \exp \cdot\left[\left(\mathrm{E}_{\mathrm{c}}-\mathrm{E}_{\mathrm{f}}\right) / \mathrm{k}_{\mathrm{B}} \mathrm{T}\right]\right.
$$

The temperature dependence of the charge carrier concentration $(n)$ is illustrated in Fig. (6) for different film thicknesses. From this figure, (n) has the order of $10^{20} \mathrm{~cm}^{-3}$ and its value increases slightly with temperature. It is found that the value of (n) has the same order of magnitude as that determined by others $[13,24]$.

The decrease of charge carriers density n with increasing film thickness, at any temperature, can be ascribed to the lattic defects, which behave as a donor level. The concentration of these defects is invesely proportinal to the film thickness. Accordingly as these defects decrease with increasing the film thickness. Accordingly as these defects decrease with increasing the film thickness, the number of free electrons per unit volume, as expected, will also decreae.

The increase of $\mathrm{n}$ with increasing temperature, at any thickness, can be attribultd to the increasing in number of electrons which were promoted across the energy gap Eventally the equilibrium density of thermally generated electrons will greatly affected by the excess of electrons due to the impuirties and thus the material will b; an intrinsic semiconductor.

\section{The Hall Coefficient Measurements :}

The Hall coefficient $\left(R_{H}\right)$ of SnTe thin films was measured at room temperatur for different film thicknesses. Fig. (3-c) illustrates the dependence of $R_{H}$ on the fill thickness, where $R_{H}$ increases with the increase of the film thickness. It was found tha 
Fig. 5 :

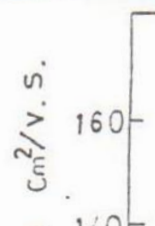

The dependence of the mobility $\left(S_{\mathrm{f}}\right)$ on the film thickness (d) at $300^{\circ} \mathrm{K}$.

2. 140
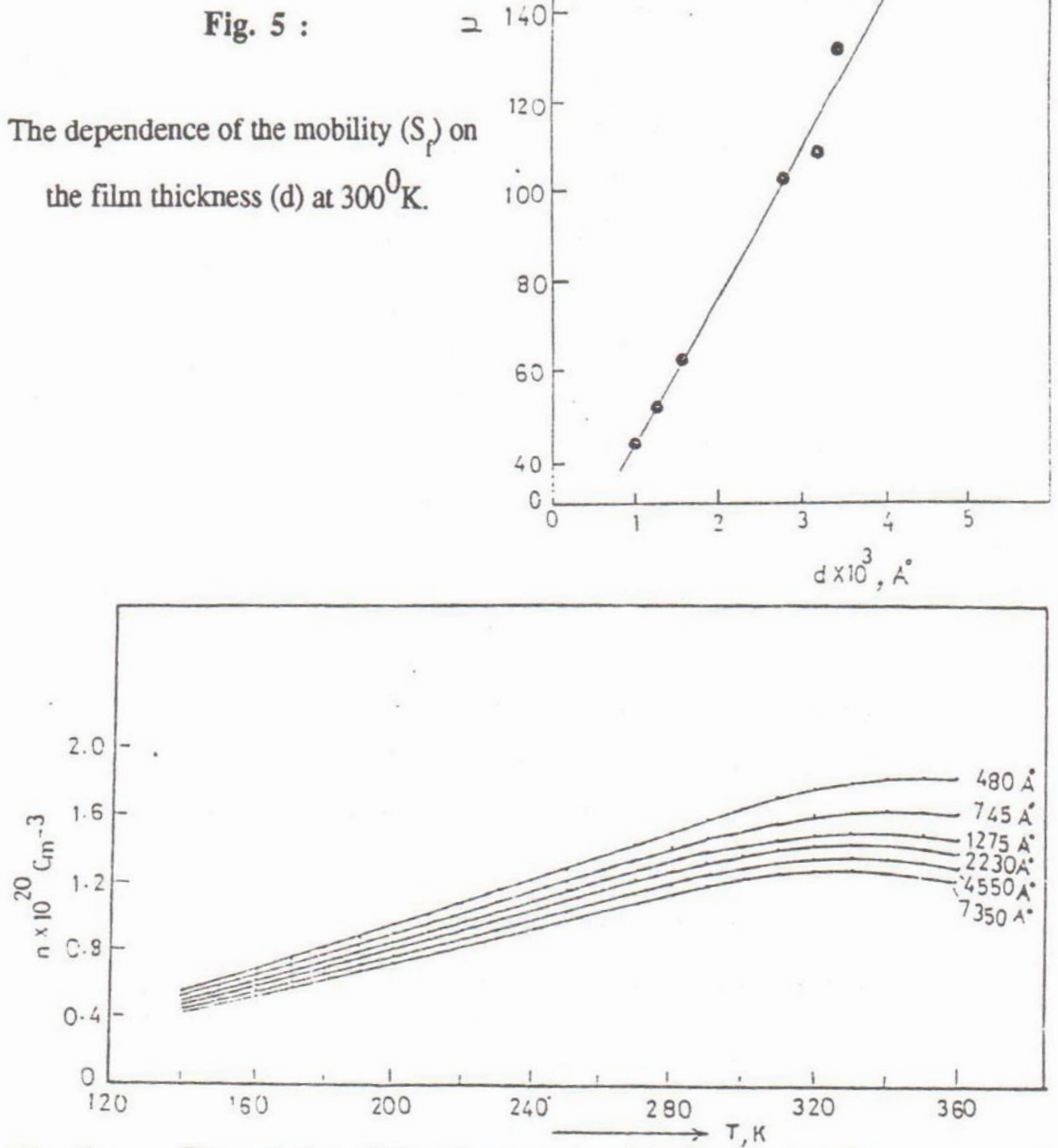

Fig. 6 : The variation of free charge carriers (n) asa function of temperature (T) for SnTe films with different thicknesses. [(n) is calculated from thermoelectric power data].

for all specimens, $R_{H}$ was negative indicating that the present films have n-type conductivity, which is in good agreement with the thermoelectric power results. Also the values of charge carrier (n) have been calculated from the Hall coefficient 
measurements and are found to be $\approx 10^{20} \mathrm{~cm}^{-3}$ (the same order of magnitude as that obtained from the thermoelectric power measurements).

The dependence of the mobility on the film thickness is shown in Fig. (5), where the mobility increases with increasing the film thickness, which could be attributed to i) the decrease of lattic defects which act as scattering centers and ii) the increase of the size of the individual crysallites leading to the decrease in the number of grain boundaries which also act as scattering centers.

Acknowledgment :

The authors gratefully acknowledge the continuous help, interest and valuable discussions of Prof. Dr. A. El-Shazly, Physics Department, Faculty of Education, Ain Shams University.

\section{REFERENCES :}

1 - $\quad$ R. F. Bis and J.R. Dixon; Phys. Rev. B-2 (1970) 1004.

2- D.M. Freik, M.A. Galushchak and A.V. Gustinov; Inorg Mater. 13 (1977) 1382.

3- V.I. Kadanov, I.A. Chernik and B.A. Efimova; Sov. Phys. Semicond. 1 (1967) 723.

4- $\quad$ R.S. Allgaier and W.S. Scanlon; Phys. Rev. 111 (1958) 1029.

5 - $\quad$ Y.W. Tung and M.L. Cohen; ibid. 180 (1969) 823.

6- $\quad$ R.S. Allgaier and B.B. Houston; ibid. B.5 (1972) 2186.

7 - A.L. Dawar, P.C. Mathur, A.O. Mohammad and O.P. Taneja; Thin Solid Films, 82 (1981) 273.

8- $\quad$ S. Santhanam and A.K. Chaudhuri; J.Phys. D : Appl. Phys. 17 (1984) 793.

9- $\quad$ Y. Ota and J. N. Zemel; J. Vac. Sci. Technol. 6 (1969) 558.

10- S. Santhanam and A.K. Chaudhuri; Physica B 115 (1983) 156.

11 - A. Goswami and R.H. Jog; Ind. J. Pure Appl. Phys. 7 (1969) 273.

12 - N.N. Zeleva; Thin Solid Films 56 (1979) 369.

13 - I.S. Athwal, Meenakshi Thaman, R.K. Bedi; J. Mater. Sci. 21 (1986) 3243.

14 - L.A. Hemstreet; Phys. Rev. 12, No. 4 (1212).

15 - A. G. Mikolaichuk, Ya. I. Dutchak and D.M. Freik; Sov. Phys. Crystallography, 13, No. 3 (1968) 490.

16 - S. Tolansky; in "Multiple-beam Interference Microscopy of Metals" Academic Press, London, (1970) 55.

17 - S. Santhanam, B.K. Samantaray and A.K. Chaudhuri; Phys. State Sol. (a), 72 (1982) 521.

18 - S. Santhanam, B.K. Samantaray and A.K. Chaudhuri; J. Phys. D. Appl. Phys. 15 (1982) 2531.

19 - P. Scherrer; Gottingen Nachrichten, 166A (1938) 16.

20 - A.A. El-Shazly, M.M. El-Nahass et al.; Egyptian J. Solids, 8, No.1 (1986) 25.

21 - A.A. El-Sharkawy, M.M. El-Oker et al.; Egyptian J.Solids, 8, No. 1 (1986) 35.

22 - A.K. Pal, P. Sen and A.K. Barua; Thin Solid Films 41 (1977) 105.

23 - A. Goswami and S.S. Koli; Proceeding of the International Symposium held at Clausthal, Gottingen 6 - 11 Sep. (1965).

24 - P.Gonzalez, J.A. Agapito and Pardo; J. Phys. C : Solid State Phys. 19 (1986) 899. 